\title{
Institutions et histoire économique
}

\author{
Alain Chatriot et Claire Lemercier \\ Chargés de recherche au CNRS, CRH-AHMOC, IHMC
}

Lors du congrès mondial d'histoire économique d'Utrecht, en août 2009, le mot « institution » ou ses dérivés était présent dans le titre de 10 des 139 sessions et de 34 des centaines de communications - et omniprésent dans les interventions des sessions plénières relatives aux « racines historiques de la pauvreté » et à la révolution industrielle. C'est peutêtre peu au vu de la fortune auto-proclamée des différents «institutionnalismes » dans les sciences sociales ${ }^{1}$. C'est en même temps beaucoup, voire trop, dans la mesure où le terme s'avère extrêmement polysémique. On relève ainsi parmi les titres des formules comme « economic modernisation in adverse institutional environments », « the benefits, costs and alternatives of British institutions », «states, institutions and development», "between networks and institutions ", " social and institutional change ", " grassroots institutions », et des références aux corporations marchandes, aux banques, au droit du travail, aux droits de propriété, tous considérés comme des «institutions ». La notion d'institution, dans l'histoire économique actuelle, peut ainsi sembler quelque peu insaisissable.

Et pourtant, si l'on compare l'historiographie actuelle, y compris en France, à celle d'il y a vingt ou trente ans, il nous semble bien que plus de chercheurs ont choisi de prendre les institutions au sérieux. Qu'entendons-nous donc par là, face au foisonnement des définitions ? Chez les seuls économistes, " institution », un terme parfois conçu comme regroupant tous les facteurs «non technologiques", peut renvoyer à une règle du jeu (souvent un élément de droit), à un acteur collectif (un service précis de l'administration, un cartel d'entreprises, voire une entreprise), à un arbitre extérieur ou un moyen de sanction (deux façons d'envisager un tribunal) ou encore à une norme de conduite, voire à une notion plus ou moins vague de «tradition » ou de « culture ». Sans choisir notre camp entre ces visions ou d'autres, issues des différentes sciences sociales, nous proposons de sortir du flou d'abord par la négative (à quelles autres notions s'oppose celle d'institutions, ou de quelles impasses propose-t-elle de sortir ?), ensuite par des considérations concrètes sur des types d'objets et des manières de les envisager qui nous semblent avoir pris une place nouvelle en histoire économique depuis quelques décennies.

Comme les titres d'Utrecht le montrent, si l'on s'intéresse aux institutions, c'est souvent en relation ou en opposition avec des conceptions simples de l'État, considéré comme un bloc et comme une contrainte à l'activité économique, du marché, vu comme un ordre spontané, voire aussi du « social », au sens où la sociabilité, la « confiance » ou d'autres aspects de la vie des acteurs considérés comme "informels » interféreraient avec leur activité économique. Cette volonté de compléter ou de dépasser un triptyque simplifié État/marché/social conduit à s'intéresser à différents types de normes et aux organisations qui les produisent ou contribuent à leur maintien ${ }^{2}$. Prendre au sérieux les institutions, c'est aussi ne pas d'emblée considérer les effets de structuration produits par les institutions comme négatifs, comme des contraintes. En réaction, les approches «institutionnalistes» en sciences sociales ont souvent une

\footnotetext{
1 Ainsi, une International Society for New Institutional Economics, définie comme « an interdisciplinary enterprise combining economics, law, organization theory, political science, sociology and anthropology to understand the institutions of social, political and commercial life. » (http://www.isnie.org/about.html) a été fondée en 1996, notamment autour de Ronald Coase, Douglass North et Oliver Williamson. Pour la France, on peut mentionner la parution récente du dossier très interdisciplinaire «Que faire des institutions ? », Tracés, 17, novembre 2009.

2 Jean-Pierre HIRSCH, Les deux rêves du commerce : entreprise et institution dans la région lilloise 1780-1860, Paris, éditions de l'EHESS, 1991, a de ce point de vue fait figure de pionnier pour l'histoire économique française, bien au-delà de la période qu'il étudiait.
} 
appréhension a priori positive d'organisations ou de règles décrites comme indispensables au fonctionnement des marchés ou comme sources d'économies de "coûts de transaction ». Nous adopterons un regard plus neutre, mettant en particulier l'accent sur la gamme des possibles institutionnels pour mettre de côté la recherche de l'optimalité économique.

Au-delà d'une définition par la négative, de quels objets est-il question ? Pour nous, il s'agit d'organisations ou de normes qui peuvent être publiques, privées ou difficiles à placer sur cette échelle, mais qui se caractérisent par une formalisation minimale qui les rend lisibles par les acteurs et permet d'identifier des frontières. En effet, une grande partie de l'activité des institutions consiste à créer des catégories : tel produit relève ou non d'une $\mathrm{AOC}$, tel marchand est ou non membre d'une corporation... Ces frontières peuvent être disputées ou transgressées, mais leur existence - et les disputes à leur sujet - contribue à structurer l'activité économique. À partir de ce constat, nous centrons ici notre propos avant tout sur des organisations (ayant peu ou prou des statuts, des membres, un budget...), ainsi que sur les normes juridiques (plutôt que sociales) que celles-ci produisent, sans adopter les définitions les plus larges des institutions. Nous prenons ainsi en compte la partie des institutions qui est directement observable dans les sources (voire qui produit ces sources) et de l'existence desquelles au moins une partie des acteurs économiques qu'elles influencent sont conscients. Cette restriction nous semble nécessaire à la fois pour mettre en évidence de nouveaux objets ou des méthodes d'étude propres à ce terrain et pour distinguer l'étude des institutions d'une plus large reconnaissance de la place du social et du politique dans l'économique. De toute façon, ce qui a changé dans l'historiographie est sans doute davantage lié à l'explicitation d'une manière de réfléchir aux institutions que la naissance d'un nouvel objet. Rien n'empêche au fond de regarder une entreprise ou une norme sociale comme une institution ; or c'est sur ces manières de voir, plutôt élaborées à partir d'organisations, que nous centrons notre propos.

Nos propres parcours de recherche nous incitent à adopter ce point de vue. Nos thèses se concentraient chacune sur une institution, en partant des archives qu'elle avait produites ; ces institutions, définies comme consultatives, présentaient l'originalité de participer à l'élaboration de politiques économiques d'État tout en n'étant pas formellement « dans l'État» de certains points de vue, notamment parce qu'elles étaient dans une certaine mesure le lieu d'expression de groupes organisés de façon privée ${ }^{3}$. Depuis, nos recherches ont eu un point de départ moins monographique, puisqu'elles sont plutôt parties de questions de politique économique - politiques de la concurrence, de la consommation, agricoles, du travail et de la formation professionnelle, modes de règlement des conflits du commerce et du travail... Mais elles ne parlent pas moins d'institutions pour autant : au contraire, ce type de questionnement permet de prendre en compte la diversité de celles qui interviennent, leurs interactions et les choix effectués entre modes de régulation possibles.

Si nous ne nous en tenons pas ici à nos propres terrains et tentons de proposer un panorama large des renouvellements historiographiques - ainsi que d'indiquer quelques pistes encore trop peu parcourues -, nous avons choisi de ne parler que de l'histoire de la période contemporaine. Cela ne signifie pas, bien au contraire, que les nouvelles approches que nous évoquons ne s'appliquent que là. Pour les périodes les plus anciennes aussi, il est bon de sortir d'une vision purement a-économique de l'administration ou purement informelle du social. Les études sur les corporations médiévales et modernes, d'une part, et sur la construction de l'État britannique, d'autre part, en particulier autour des questions de fiscalité et de droits de propriété ${ }^{4}$, ont été au coeur d'importants débats internationaux impliquant économistes et

3 Alain Chatriot, La démocratie sociale à la française. L'expérience du Conseil National Economique, 19241940, Paris, La Découverte, 2002. Claire Lemercier, Un si discret pouvoir. Aux origines de la Chambre de commerce de Paris 1803-1853, Paris, La Découverte, 2003.

4 Pour un bilan récent sur les corporations, voir Jan Lucassen, Tine De Moor, Jan Luiten van Zanden, « he Return of the Guilds: Towards a Global History of the Guilds in Pre-industrial Times », International Review of Social History, Volume 53, Issue S16, December 2008, p. 5-18; sur la France, Steven L. KaPlan, La fin des 
historiens - à tel point que, du côté des économistes, les cas historiques traités par les approches institutionnalistes datent plus souvent de l'avant que de l'après-1800. Il ne nous en semble que plus intéressant de discuter des recherches réalisées sur les deux derniers siècles, généralement considérés comme l'âge d'or du libéralisme débridé (pour le XIx ${ }^{\mathrm{e}}$ ) et/ou de la bureaucratisation. Montrer qu'il y a pourtant, pour ces périodes aussi, une place pour une étude des institutions entendues autrement que comme entraves au marché - y compris de " corps intermédiaires » auxquels on gagnerait souvent à appliquer les approches développées à propos des corporations anciennes ${ }^{5}$ - n'en est que plus nécessaire.

Dans ce cadre, nous rappelons à quels changements plus généraux de la discipline historique, mais aussi d'autres sciences sociales répond l'émergence d'un intérêt pour les institutions en histoire économique. Puis nous précisons ce qu'implique selon nous ce nouveau regard en termes de rapport aux sources et aux méthodes, nous dégageons des questionnements prioritaires et évoquons quelques objets dont cette approche a particulièrement renouvelé l'étude.

\section{Pourquoi les institutions ?}

\section{Des évolutions propres à l'histoire...}

Avec, notamment, l'histoire des entreprises, l'étude des institutions est une des approches de l'histoire économique qui s'est développée depuis quelques décennies aux dépens d'une part d'une histoire macroéconomique quantitative et d'autre part d'une histoire économique et sociale à visée globale, mais saisie dans le cadre d'un secteur et/ou d'une région. Comme les entreprises, les institutions sont des productrices de fonds d'archives, de plus souvent conservés et inventoriés lorsqu'elles sont publiques : or la tentation de la monographie fermement centrée sur un fonds bien délimité et de taille raisonnable (et, dans le pire des cas, limitée à lui) a certainement été encouragée par le format des thèses " nouveau régime ». En outre, la vogue de la prosopographie et notamment de l'« histoire des élites $»^{6}$, qui ne semble pas se démentir depuis les années 1970 et qui a également donné naissance à nombre d'études de chefs d'entreprise, incite à aborder les institutions du point de vue des biographies collectives : il est ainsi plus facile de circonscrire un corpus bien identifié de membres présents sur des listes plus ou moins officielles que de décider comment on va définir « les patrons » ou « les inventeurs ».

Ces raisons très pratiques de choix de terrains d'étude sont intimement liées à d'autres qui relèvent plus de l'évolution des débats historiographiques. Ainsi, l'intérêt pour les institutions en tant que productrices de sources est intimement lié à la prise de conscience du caractère construit des catégories statistiques, notamment dans le cadre des critiques de l'histoire quantitative première manière, et plus généralement des catégories - socioprofessionnelles, juridiques... - utilisées pour décrire les fonctionnements économiques et agir sur eux ${ }^{7}$. Des

corporations, Paris, Fayard, 2001, Stephen L. Kaplan, Philippe Minard (dir.), La France, malade du corporatisme? XVIII $-X X^{e}$ siècles, Paris, Belin, 2004. Douglass C. North, Robert Paul Thомаs, The Rise of the Western World: A New Economic History. New York, Cambridge University Press, 1973.

5 Alain Chatriot, Claire Lemercier, «Les corps intermédiaires », in Vincent Duclert, Christophe Prochasson (dir.), Dictionnaire critique de la République, Paris, Flammarion, 2002, p. 691-698.

6 Christophe Charle, Jean Nagle, Marc Perrichet, Michel Richard, Denis Woronoff, Prosopographie des élites françaises $\left(X V I^{e}-X X^{e}\right.$ siècles). Guide de recherche, Paris, CNRS-IHMC, 1980.

7 L'attention nouvelle à cette construction institutionnelle des catégories dépasse bien sûr le domaine de l'histoire économique. D'un point de vue général, voir Mary Douglas, Comment pensent les institutions, Paris, La Découverte, 1999 (1986). En France et en histoire économique et sociale, la parution de Alain Desrosières, Laurent ThÉvenot, Les catégories socioprofessionnelles, Paris, La Découverte, 1988 a représenté une étape importante pour cette prise de conscience. 
recherches centrées au départ sur le travail et le chômage, l'agriculture ou les migrations ont ainsi bifurqué, provisoirement ou définitivement, vers l'étude des organismes qui avaient produit des chiffres ou d'autres types de descriptions de ces phénomènes à l'époque étudiée, menant parfois à une véritable histoire des savoirs et/ou des politiques économiques ${ }^{8}$.

$\mathrm{Si}$ les institutions produisent ainsi des catégories qui mettent en ordre le réel, elles sont également impliquées - c'est même une des définitions que l'on peut donner d'elles - dans la production de règles qu'un certain nombre d'acteurs économiques sont censés respecter, et/ou dans la mise en application de ces règles. Lorsque l'on pense que de telles règles ne sont en général pas nécessaires, que les marchés, par exemple, s'auto-régulent, ou au contraire qu'elles sont quasiment immuables et suivies tout naturellement par les acteurs, comme dans les schémas culturalistes les plus simples ${ }^{9}$, il n'est guère utile de se pencher sur les institutions. $\mathrm{Au}$ contraire, les débats autour de la micro-histoire, en particulier ${ }^{10}$, se situant eux-même bien souvent dans une discussion des travaux de Michel Foucault, ont mis l'accent depuis les années 1980 sur la nécessité de dépasser ces deux visions, de travailler par exemple sur les exceptions qui mettent en évidence, par leur existence même, à la fois la règle et les marges de manoeuvre autour d'elle. Les institutions se construisent et se délitent, il existe des moments de choix entre plusieurs possibles, des occasions de critique et de réforme ; les acteurs économiques peuvent jouer sur des règles concurrentes ou des écarts à la règle, interpréter, tricher ou transgresser, sans pour autant, au moins un temps, faire disparaître le poids propre aux institutions ${ }^{11}$. Une partie des débats historiographiques plus généraux sur la notion d'agency et le déterminisme a pu ainsi mener à une prise en compte diachronique et nuancée des institutions qui ne se limite pas à leurs attributions "sur le papier » mais essaie de reconstituer des pratiques, que ce soient celle de membres d'organisations ou d'autres acteurs confrontés à des règles.

Même si l'histoire des institutions ne se limite pas aux plus officielles d'entre elles, ces évolutions ont notamment fait voir sous un nouveau jour l'histoire de l'État et celle du droit, longtemps considérées comme antagonistes de l'histoire économique et sociale. Une histoire sociale de l'administration s'est développée contre la vision de «l'État» comme un bloc impersonnel ${ }^{12}$; les débats des modernistes sur la construction de «l'État moderne » ou sur l'anachronisme éventuel de la notion de "politique économique $»^{13}$ ont permis de souligner que le statut de fonctionnaire, la notion de service public ou l'existence d'un ministère de l'Économie, par exemple, étaient loin d'aller de soi, et les interrogations sur l'histoire de tels

8 Comme témoignage de certaines de ces trajectoires, voir par exemple Jean Luciani (dir.), Histoire de l'Office du Travail (1890-1914), Paris, Syros, 1992; Alessandro Stanziani, L'économie en révolution. Le cas russe, 1870-1930, Paris, Albin Michel, 1998 ; Paul-André Rosental, L’intelligence démographique. Sciences et politiques des populations en France (1930-1960), Paris, Odile Jacob, 2003 ; Béatrice Touchelay et Patrice Verheyde (dir.), La genèse de la décision. Chiffres publics, chiffres privés dans la France du XXe siècle, Pompignac près Bordeaux, Bière, 2009.

9 Ce que Mark Granovetter a finement décrit comme les visions « sous-socialisée » et «sur-socialisée » de l'économie. Mark Granovetter, "Economic Action and Social Structure: The Problem of Embeddedness", American Journal of Sociology, 91(November 1985): 481-510.

10 Jacques REVEL, «L'institution et le social », in Bernard LePETIT (dir.), Les formes de l'expérience. Une autre histoire sociale, Paris, Albin Michel, 1995, p. 63-84.

11 Voir notamment Nicolas Delalande, Consentement et résistances à l'impôt : l'Etat, les citoyens et le problème de la confiance sous la III République, thèse de doctorat d'histoire, sous la dir. de C. Charle, Université Paris 1, 2009. La notion de jeu sur des « dénivellations réglementaires » a ainsi été bien exposée par Philippe Minard. Voir par exemple Philippe MinARD, «Les formes de régulation du travail en France et en Angleterre au XVIII ${ }^{e}$ siècle : une enquête en cours », Cahiers de Framespa, 1, 2006, non paginé (revue en ligne). 12 Marc Olivier Baruch, Vincent Duclert (dir.), Serviteurs de l'Etat, une histoire politique de l'administration française 1875-1945, Paris, La Découverte, 2000.

13 Philippe Minard, « État et économie en France après la Révolution ». Historiens et géographes, ${ }^{\circ} 378,2002$, p. $195-201$. 
instruments ont maintenant cours également en histoire contemporaine ${ }^{14}$. Plus récemment, surtout dans les années 2000, cette attention portée aux pratiques administratives concrètes a été complétée par des interrogations sur le droit, c'est-à-dire à la fois sur la construction conflictuelle de catégories juridiques comme celles de brevet, d'appellation d'origine contrôlée ou de concurrence déloyale et sur la mobilisation de la justice (ou d'autres formes de règlement des conflits) par les acteurs économiques ${ }^{15}$. Enfin, si les bureaux ministériels, les administrations locales, voire les institutions consultatives et les tribunaux commencent ainsi à être mieux explorés, on peut s'interroger sur le paradoxal désintérêt pour les aspects parlementaires de l'histoire des normes économiques, des débats à la tribune au travail des commissions ${ }^{16}$ - peut-être lié à la volonté de souligner que la loi ne se prépare pas seulement là où elle se vote, ou bien à la monopolisation de ce type d'études par l'histoire politique.

Un autre phénomène lié à des changements historiographiques généraux a permis de mettre l'accent sur certaines institutions jouant un rôle marquant pour les questions économiques. De nouvelles formes de demandes sociales et de désirs de commémoration ont eu des conséquences sur le travail des historiens. Si le phénomène a surtout concerné, pour l'histoire économique, l'histoire des entreprises, de nombreux ministères ont aussi créé, à la suite de celui de la Sécurité sociale, des comités d'histoire. Ces comités, en regroupant hauts fonctionnaires et historiens, ont organisé assez souvent la constitution d'archives orales, l'élaboration de guides de sources et le soutien à des recherches historiques. Ils ont parfois permis que des anniversaires ne se résument pas à des " grands messes » médiatiques, mais aident aussi à faire progresser la connaissance sur des objets trop peu étudiés ${ }^{17}$.

Le découpage administratif des ministères pourrait faire penser que de tels apports n'ont concerné que l'histoire de l'Etat. Or, ce sont les ministères liés au travail, à la protection sociale, à l'économie et aux finances qui ont été en pointe dans l'ouverture aux historiens, en ne s'intéressant pas qu'à une histoire strictement interne. Avec d'importants moyens financiers, le Comité pour l'histoire économique et financière de la France a permis de nombreuses publications qui ont éclairé bon nombre des institutions économiques françaises, même si ces études généralement monographiques ont parfois peu mis en question leur lien à des fonds d'archives exclusifs. Dans le même temps, le Comité, maintenant associé à l'Institut de la gestion publique et du développement économique, aide à l'émergence de travaux novateurs, comme ceux nés de la confrontation entre historiens, politistes et juristes sur les questions du système financier et du droit budgétaire et comptable ${ }^{18}$.

De grands établissements financiers ont aussi encouragé les initiatives des historiens, qui ont ainsi pu proposer des approches de longue durée souvent en prise avec des questions

14 Voir les dossiers «Le service public, l'économie, la République 1780-1960 », Revue d'histoire moderne et contemporaine, 52, 3, 2005 et «Bientôt privés de services publics », Regards croisés sur l'économie, 2, septembre 2007.

15 Voir notamment Alessandro Stanziani (dir.), Dictionnaire historique de l'économie-droit, XVIII ${ }^{e}-X X^{e}$ siècles, Paris, LGDJ, 2007. Les guides des sources et bibliographies élaborés par Jean-Claude Farcy fournissent une aide inestimable aux recherches dans ce domaine: Guide des archives judiciaires et pénitentiaires (1800-1958), Paris, CNRS éditions, 1992 et Deux siècles d'histoire de la Justice en France. Notices bibliographiques, Paris, CNRS éditions, 1996, repris et enrichis sur http://www.criminocorpus.cnrs.fr

16 Voir, à propos de débats sur les marchés publics, Hélène LemesLe, « Apprendre le travail parlementaire et construire la séparation des pouvoirs dans les années $1870 »$, Revue d'histoire du XIX siècle, 35, 2007, p. 121139 et pour une période plus récente: Pierre LASCOUMES, «Les compromis parlementaires, combinaisons de surpolitisation et de sous-politisation. L'adoption des lois de réforme du Code pénal (décembre 1992) et de création du PACS (novembre 1999) », Revue française de science politique, 59, 2009-3, p. 455-478.

17 On pense ici aux mobilisations scientifiques rendues possibles dans le cadre du centenaire du ministère du Travail en 2006 : Alain Снатriot, Odile Join-Lambert, Vincent Viet (dir.), Les Politiques du Travail (19062006). Acteurs, institutions, réseaux, Rennes, Presses universitaires de Rennes, 2006.

18 Philippe Beż̀s, Florence Descamps, Sébastien Kott, Lucile Tallineau (dir.), La mise en place du système financier public 1815-1914. Elaborations et pratiques du droit budgétaire et comptable au XIX siècle, Paris, CHEFF, 2010. 
d'actualité : du rôle des banques centrales ${ }^{19}$ à l'action d'un établissement comme la Caisse des dépôts et consignations sous l'Occupation ${ }^{20}$. Au-delà d'une histoire des entreprises applicable à l'identique dans d'autres secteurs, l'histoire bancaire a ainsi été également renouvelée par une approche institutionnelle. Ces demandes ne se résument pas au seul secteur financier: d'autres institutions traitant de questions économiques ont pu susciter, par exemple, un meilleur inventaire de leurs archives déposées dans des archives publiques ${ }^{21}$ - même si c'est parfois aussi l'initiative d'archivistes, sans demande institutionnelle, qui a permis la mise en valeur de tels fonds ${ }^{22}$. Même à l'échelle locale ou régionale, les institutions consultatives peuvent s'intéresser à leur histoire, inventorier leurs archives de façon exemplaire ou mobiliser les spécialistes de sciences sociales ${ }^{23}$.

\section{... ou partagées avec d'autres sciences sociales}

Si certaines de ces évolutions sont propres à l'histoire, beaucoup, comme l'attention portée au caractère construit des catégories, aux jeux autour des règles ou aux usages du droit, sont largement partagées avec d'autres sciences sociales. De très nombreuses variétés $\mathrm{d}^{\prime}$ « institutionnalismes », décrites comme telles par leurs promoteurs et donc plus clairement reconnues et définies qu'en histoire, s'y sont développées depuis les années 1980. Il ne s'agit pas ici de passer en revue chacun de ces courants, mais d'indiquer quelques pistes bibliographiques tout en réfléchissant à ce qu'ils ont apporté, ou pourraient apporter, aux historiens de l'économie.

Rares sont les économistes, tout d'abord, à s'en tenir aujourd'hui à une vision totalement ainstitutionnelle du monde, où les marchés s'auto-réguleraient en vertu d'une rationalité parfaite. Alors qu'une première génération d'« institutionnalistes », dans la première moitié du $\mathrm{xx}^{\mathrm{e}}$ siècle, s'intéressait au droit et aux institutions dans le cadre d'une description des fonctionnements concrets de l'économie qui prenait en compte ses fondements «extraéconomiques $»^{24}$, les nouveaux institutionnalismes réintègrent les institutions à l'intérieur même du raisonnement économique. Ils partent souvent d'une réflexion théorique sur les insuffisances des marchés (information imparfaite ou asymétrique, que ce soit sur les qualités des produits ou le comportement des acteurs) ou sur les coûts de transaction (par exemple ceux liés à l'établissement de contrats détaillés) pour expliquer qu'il existe un besoin d'institutions - dans ce cas souvent décrit comme une sorte de pis-aller. Cette approche conduit fréquemment à adopter un point de vue normatif, en particulier dans le courant law and economics : on cherche alors à mesurer l'efficacité de différentes organisations ou règles juridiques possibles, soit sur la base de modèles déductifs comme ceux de la théorie des jeux, soit à partir de l'observation de données à la fois institutionnelles et économiques, actuelles ou

19 Olivier Feiertag, Michel Margairaz (dir.), Politiques et pratiques des banques d'émission en Europe, XVII $X X^{e}$ siècles : le bicentenaire de la Banque de France dans la perspective de l'identité monétaire européenne, Paris, Albin Michel, 2003.

20 Alya Aglan, Michel Margairaz, Philippe Verheyde (dir.), La Caisse des dépôts et consignations, la Seconde Guerre mondiale et le XXe siècle, Paris, Albin Michel, 2003.

21 Archives nationales, Conseil économique, social et environnemental, Le Conseil économique 1946-1960, 2009, 233 p., direction scientifique Alain Снатвіот et Christian Oppeтiт pour le répertoire numérique détaillé.

22 Brigitte LAINÉ, Le conseil de prud'hommes du département de la Seine : 1844-1940 (1762-1971), Paris, Archives de Paris, 2005 ; Brigitte LaINÉ, Juridiction consulaire 1563-1792, Tribunal de commerce 1792-1997, Paris, Direction des Services d'Archives de Paris, 2009.

23 Olivier CotTarel, Répertoire numérique des séries anciennes (archives collectées de 1803 à 1986), Paris, Chambre de commerce et d'industrie de Paris, 1996 ; Patrick Moquay, Jacques PALARD, La société régionale en dialogue : le Conseil économique et social d'Aquitaine 1974-1989 : l'innovation apprivoisée, Bordeaux, Conseil économique et social régional d'Aquitaine, éditions confluences, 2009.

24 Alain GuÉRY, «Propriété, droit et institution dans l'institutionnalisme américain », Cahiers d'économie politique, 40-41, 2001, p. 9-38. 
passées, traitées par l'économétrie. En outre, le constat de la diversité des institutions présentes dans la réalité, et en particulier de la survie de certaines, considérées comme inefficaces ou en tout cas non optimales par ces approches, a conduit à des interrogations sur les mécanismes du changement institutionnel, souvent formulées en termes de path dependence : l'accent est alors mis sur ce qui peut faire qu'une institution, même inefficace, se maintient.

Certains aspects de ces travaux heurtent frontalement les pratiques historiennes, de par leur côté très normatif ou leur ignorance de la diversité des acteurs, des conflits politiques ou des rapports de forces. C'est par exemple le cas du courant law and growth, très influent dans les institutions internationales, qui tente de démontrer l'infériorité fondamentale des systèmes dérivés d'une supposée "tradition juridique » française. Mais la volonté de démontrer les insuffisances de cette approche a justement stimulé d'importants travaux d'histoire économique qui soulignent l'importance des circulations internationales de modèles institutionnels, l'inexistence de «traditions » linéaires ou encore les divergences entre lettre des lois et pratiques autorisées par les tribunaux ${ }^{25}$.

D'autres courants entretiennent, ou pourraient entretenir, un dialogue plus fécond avec les historiens. Ainsi, certains concepts de l'économie des conventions ${ }^{26}$ et de l'économie de la régulation, écoles hétérodoxes particulièrement présentes en France, ont été utilisés par des historiens de l'économie. La notion de convention de qualité permet notamment de souligner le caractère construit et, plus précisément, négocié de la qualité des biens ${ }^{27}$. La description de la diversité des "modèles productifs » par l'école de la régulation a plutôt intéressé une histoire des entreprises sensible aux questions de "gouvernance»; l'extension de ces interrogations comparatives aux arrangements institutionnels à l'échelle d'une société entière, qui pourrait être pertinente pour l'histoire des institutions économiques, ne semble pas pour l'heure avoir eu le même écho ${ }^{28}$. Enfin, certains économistes a priori plus orthodoxes utilisent la théorie des jeux, partant donc d'une approche des transactions entre individus, mais en l'adossant à des études de cas historiques détaillées, à une vision évolutionniste des institutions et en prenant en compte le fait qu'il y a rarement un seul équilibre possible. Cela permet d'explorer efficacement les divers arrangements institutionnels possibles, notamment les formes complexes d'« ordre privé », qui ont en réalité leurs propres institutions ${ }^{29}$. Ces approches, encore peu connues et surtout mobilisées par les historiens français, ont le mérite d'insister sur les mécanismes qui font, ou non, qu'une règle est appliquée par les acteurs (ce qui renvoie aux facteurs sociaux et culturels du fonctionnement des institutions, voire à ceux qui permettent de se passer d'institutions étatiques, comme les effets de réputation) et sur la diversité des formes de régulation possibles, qui ne se réduisent pas au pur marché d'une part et à la législation centralisée et implacable de l'autre.

Si l'histoire du droit, en France, continue à ne guère se préoccuper d'aspects économiques ou, lorsqu'elle le fait, à en rester à une étude du contenu des lois, de la doctrine ou de la

25 Pour une présentation de ces travaux et débats, voir Claire Lemercier, « Napoléon contre la croissance ? À propos de droit, d'économie et d'histoire », La vie des idées, 21 novembre 2008.

26 Voir notamment Christian Bessy, Olivier Favereau, «Institutions et économie des conventions », Cahiers d'économie politique, 44, 2003, p. 119-164.

27 Alessandro Stanziani (dir.), La qualité des produits en France (XиII ${ }^{e}-X X^{e}$ siècles), Paris, Belin, 2003 ; Alessandro Stanziani, Histoire de la qualité alimentaire XIX ${ }^{e}-X X^{e}$ siècle, Paris, Le Seuil, 2005.

28 Robert Boyer, Michel Freyssenet, Les modèles productifs, Paris, La Découverte, 2000 et Robert Boyer, J. Rogers Holuingsworth (eds.), Contemporary Capitalism : The Embeddedness of Institutions, Cambridge University Press, Cambridge, 1997.

29 Masahiko Аокі, Fondements d'une analyse institutionnelle comparée, Paris, Albin Michel, 2006 (2001) (voir aussi Hervé Defalvard, « La théorie aokienne des institutions à l'épreuve de la loi de 1841 sur le travail des enfants ", Document de travail du Centre d'études sur l'emploi, n² 22, mars 2003); Avner GreIF, "Qu'est-ce que l'analyse institutionnelle? », Tracés, 17, 2009, p. 181-210 (2006), discuté par Robert BOYER, « Historiens et économistes face à l'émergence des institutions du marché », Annales HSS, 3, 2009, p. 665-693. 
jurisprudence des cours les plus élevées, les renouvellements de la sociologie du droit - telle que pratiquée par le courant law and society, qui compte en réalité plus de juristes que de sociologues - proposent des approches et des objets propres à enrichir l'agenda de l'histoire des institutions. Certes, les appels à une sociologie du droit dans l'activité économique restent encore assez largement programmatiques ${ }^{30}$; plus précisément, leurs premiers résultats marquants touchent surtout au monde du travail, avec par exemple des études des effets concrets des réglementations contre la discrimination ou en matière de sécurité, de la réduction du temps de travail ou du recours aux prud'hommes. Toutefois, rien n'empêche d'appliquer à d'autres aspects de l'économie des interrogations générales sur le « droit en action » plutôt que « dans les livres » et notamment sur la conscience que les acteurs ont du droit et sur sa présence dans la vie quotidienne, bien au-delà des ministères, des Parlements ou des tribunaux - sous forme de formulaires, d'affiches ou d'étiquettes par exemple ${ }^{31}$.

Adopter cette vision plus large et sociologique du droit ne dispense en revanche pas de porter attention aux techniques spécifiques à cette discipline, qui s'avèrent nécessaires pour comprendre les usages possibles de sources comme les recueils de jurisprudence, les thèses de droit du passé ou les archives des tribunaux ${ }^{32}$. De premiers travaux issus de rencontres interdisciplinaires montrent ce que cette prise en compte des procédures et de la façon dont les acteurs peuvent les utiliser - par exemple en choisissant de mener un procès au civil ou au pénal, d'utiliser la faillite ou la liquidation judiciaire... - peut apporter à l'histoire économique $^{33}$. Sans se transformer en juristes, les historiens de l'économie peuvent devenir plus attentifs à l'environnement juridique qui était celui des acteurs du passé (jusqu'aux ouvriers, agriculteurs, petits commerçants...) et à l'appréhension qu'ils pouvaient en avoir, par exemple sur la base de manuels de vulgarisation ou de la presse, des sources encore très peu exploitées sur ces questions.

Une autre science sociale a connu une vague néo-institutionnaliste au cours des vingt dernières années : la science politique ${ }^{34}$. Ces propositions ont surtout concerné l'approche des politiques publiques, en y apportant une attention plus spécifique aux fonctionnements des institutions dans le processus d'action publique. Cela revient à souligner que les choix politiques sont influencés directement par la structure des institutions politiques qui définissent les « règles du jeu ». Les politistes distinguent habituellement trois branches du néo-institutionnalisme, aux hypothèses de travail assez différentes : l'approche sociologique, héritée de la sociologie des organisations, l'approche en termes de choix rationnel, parente des analyses économiques, et une approche plus historique. Cette dernière vise à prendre en compte les institutions comme l'une des variables d'explication de l'évolution des sociétés politiques ; souvent en utilisant la notion de path dependence. Ainsi les institutions saisies par la science politique ne se résument plus aux simples descriptions parfois proposées par

30 Richard Swedberg, «The Case of an Economic Sociology of Law », Theory \& Society, 32, 2003, p. 1-37; Lauren Edelman, Robyn Stryker, "A Sociological Perspective on Law and the Economy », in Neil Smelser, Richard Swedberg (eds.), The Handbook of Economic Sociology, Princeton: Princeton University Press, 2005 (1994), p. 527-551.

31 Jérôme PÉLIsse, «A-t-on conscience du droit ? Autour des Legal Consciousness Studies », Genèses, 59, 2005 p. 114-130. Sur les usages du droit par des groupes d'intérêts, voir notamment Hélène Michel, La cause des propriétaires. Etat et propriété en France, fin XIX $X^{e}-X X^{e}$ siècle, Paris, Belin, 2006.

32 Pour un bel exemple de connaissance fine de la procédure mise au service d'une histoire économique - en histoire moderne - voir Amalia D. Kessler, A Revolution in Commerce: The Parisian Merchant Court and the Rise of Commercial Society in Eighteenth-Century France, Londres, New Haven, Yale University Press, 2007. 33 Alessandro Stanziani (dir.), Dictionnaire..., op. cit.; Gérard Beaur, Hubert Bonin, Claire Lemercier dir., Fraude, contrefaçon et contrebande, de l'Antiquité à nos jours, Genève, Droz, 2006 ; Pierre-Cyrille HaUtcoeur (dir.), « Justice commerciale et histoire économique. Enjeux et mesures », Histoire \& Mesure, XXIII-1, 2008. 34 Pour une synthèse : Sven Steinmo, « Néo-institutionnalismes », in Laurie Boussaguet, Sophie Jacquot, Pauline Ravinet (dir.), Dictionnaire des politiques publiques, Paris, Presses de SciencesPo, 2004, p. $290-297$. 
certains juristes. Au contraire, les formes institutionnelles elles-mêmes sont des objets d'étude dans les processus de réforme administrative tout au long du $\mathrm{XX}^{\mathrm{e}}$ siècle $^{35}$.

Résumées par les «trois $\mathrm{i}$ », pour «idées, institutions, intérêts ${ }^{36}$, ces approches des politistes font des institutions un des observatoires privilégiés des mobilisations collectives et de l'action de l'Etat. Cette grille d'analyse permet de combiner plusieurs hypothèses théoriques à propos des processus étudiés.

Un bon exemple de ce type d'approche, concernant l'histoire économique, concerne la régulation du marché du vin en France. Que ce soit dans le cadre d'une approche sociologique sur la Bourgogne ${ }^{37}$ ou de politistes sur le Bordelais ${ }^{38}$, ces travaux placent au cœur de l'observation de la construction des marchés économiques différentes institutions. Alors que, longtemps, les seuls travaux de sciences sociales sur les expériences viticoles françaises étaient dus à des géographes - la géographie économique apportant d'ailleurs parfois sa richesse d'analyse, dépassant une description plate de terroirs -, l'apport des autres sciences sociales a aidé à percevoir le rôle de beaucoup d'acteurs collectifs et d'institutions longtemps peu étudiés. Les syndicats et leur confrontation à l'État par le biais des organisations professionnelles chargées du suivi des appellations d'origine contrôlée doivent ainsi être pris en compte pour comprendre l'évolution du monde viticole français au $\mathrm{XX}^{\mathrm{e}}$ siècle. On retrouve, à travers ce marché très spécifique du vin, des configurations qui évoquent ce que le sociologue Lucien Karpik nomme « l'économie des singularités " ${ }^{39}$.

D'autres « institutionnalismes» se sont en effet, pour finir, développés en sociologie économique, souvent en dialogue à la fois avec ceux des politistes et des économistes ${ }^{40}$. Ils mettent en particulier l'accent sur le fait que le marché lui-même a besoin, pour fonctionner, d'institutions, pas seulement comme fondements un peu lointains (comme la monnaie ou les droits de propriété, pris en compte par les économistes institutionnalistes), mais comme moyens tout à fait concrets d'obtenir l'ajustement entre offre et demande par un prix. Les études sociologiques ou ethnographiques de marchés et de transactions contemporains ${ }^{41}$ ont ici largement dialogué avec les travaux d'historiens, notamment sur les institutions de mesure ou de certification qui rendent l'échange possible ${ }^{42}$.

\section{Sources et méthodes}

Dans ce contexte interdisciplinaire, l'apport des historiens réside sans doute dans l'attention portée aux temporalités, aux relations entre discours et pratiques et tout simplement aux archives. L'approche des institutions que nous présentons ici, centrée sur des études d'organisations plus que de normes (mais étudier ces dernières renvoie souvent à différentes

35 Alain Снатriot, «Les Offices en France sous la troisième République. Une réforme incertaine de l'Administration ", Revue française d'administration publique, 120, 2006, p. 635-650; Philippe BEZES, Réinventer l'Etat. Les réformes de l'administration française (1962-2008), Paris, PUF, 2009.

36 Yves Surel, «Trois I », in L. Boussaguet, S. Jacquot, P. Ravinet (dir.), Dictionnaire ..., op. cit., p. 452-459.

37 Gilles LAFERTÉ, La Bourgogne et ses vins : image d'origine contrôlée, Paris, Belin, 2006.

38 Andy Smith, Jacques de Maillard, Olivier Costa, Vin et politique. Bordeaux, la France, la mondialisation, Paris, Sciences Po Les Presses, 2007.

39 Lucien KaRPIK, L'économie des singularités, Paris, Gallimard, 2007.

40 Victor NeE, "The New Institutionalism in Economics and Sociology" in Neil Smelser, Richard SwedBerg (eds.), The Handbook of Economic Sociology, Princeton: Princeton University Press, 2005 (1994), p. 19-45.

41 En particulier Marie-France GARCIA-PARPET, « La construction sociale d'un marché parfait : le marché au cadran de Fontaine-en-Sologne », Actes de la Recherche en Sciences Sociales, 65, 1986, p. 2-13. Plus généralement, voir Caroline Dufy, Florence Weber, L'ethnographie économique, Paris, La Découverte, 2007 et Pierre Françols, Sociologie des marchés, Paris, Armand Colin, 2008.

42 Dominique Margaraz, «Les bureaux de poids public et l'organisation de l'espace marchand », Revue du Nord, t. 85, n 352, octobre-décembre 2003, p. 845-862; Dominique Margairaz, Philippe Minard (dir.), « Le marché dans son histoire », Revue de synthèse, 127-2, 2006. 
organisations...), n'est pas propre à l'histoire économique, mais elle y a été pratiquée dans un nombre croissant de travaux depuis une quinzaine d'années.

\section{Les échelles temporelles et spatiales de l'institution}

Si l'histoire renouvelée des institutions met l'accent sur les pratiques dans le sens où elle ne se limite pas à une approche par les textes de droit, elle n'exclut pas pour autant, au contraire, tout intérêt pour les discours de l'institution, ni pour les discours à son propos. Il s'agit en particulier de s'interroger sur la légitimation et la construction d'identité des institutions, de façon diachronique et en accordant toute l'attention nécessaire aux moments de création et de controverses. Le terme, toujours quelque peu piégé, d' «identité » d'une institution renvoie pour nous à quelque chose qui doit être rendu observable dans les sources : on peut ainsi l'envisager sur la base de l'étude de l'implication de ses membres (importante ou non, exclusive ou non), ou encore du vocabulaire qu'ils utilisent. Par exemple, pour comprendre dans quelle mesure une institution est l' " héritière » d'une autre - un élément important d' «identité »-, on peut à la fois se demander si ceux qui l'ont créée l'ont pensée explicitement comme telle, si des pratiques matérielles, des bureaux, des formulaires identiques y sont utilisés, si des membres ont circulé de l'une à l'autre ou ont été formés dans les mêmes lieux, si les mêmes acteurs ou autres institutions y font appel, si les discours produits par l'institution comme collectif se réfèrent explicitement à un modèle antérieur ou encore emploient les mêmes mots...

Plus généralement, étudier la création d'une institution oblige à s'interroger sur des temporalités différentes : celle des élaborations doctrinales (souvent contradictoires), puis celle des expériences inspiratrices et des problématisations plus précises, celle de la décision de création elle-même et enfin celle d'une création continue, qui se prolonge sous forme d'installation dans la durée (d'institutionnalisation) et qui peut fortement transformer une institution. Analyser de tels moments impose de redécouvrir les autres solutions qui étaient considérées comme possibles pour le même problème et de repérer la diversité des lieux de débats autour d'un tel projet ${ }^{43}$.

Bien évidemment, pister la présence, et le cas échéant l'image, d'une institution dans des sources qu'elle n'a pas produites, voire qui a priori ne sont pas spécialement censées parler d'elle, est tout aussi crucial. Trop de monographies en restent à une étude interne, pour des raisons de choix d'archives, qui peuvent amener à surestimer l' "influence» ou la «centralité », quelle que soit la manière de les définir, de l'institution étudiée. Pour comprendre comment des acteurs extérieurs se la représentent - ce qu'ils pensent de ses membres, des intérêts qu'elle défend, de son influence, etc. -, il faut à la fois varier les points d'entrée du point de vue des archives, en partant par exemple de questions transversales et de fonds totalement extérieurs, et être attentif, dans les archives mêmes de l'institutions, aux traces de circulations et d'interactions qui peuvent permettre de comprendre par exemple à quel stade elle se situe dans des processus de décision et qui choisit ou non de s'adresser à elle. Ainsi, Marie-Emmanuelle Chessel, étudiant une association de consommateurs impliquée dans la campagne pour une loi sur le travail du dimanche, peut à la fois discuter de la centralité de cette campagne pour l'association, mais aussi, en adoptant d'autres entrées archivistiques, du poids - tout relatif - de cette organisation particulière dans un mouvement d'opinion, puis dans les mécanismes d'élaboration et d'application de la $1 \mathrm{li}^{44}$.

43 Pour des exemples de telles analyses, voir Alain Chatriot, Claire Lemercier, « Une histoire des pratiques consultatives de l'Etat », in Michel OfFerle, Henry Rousso dir., La fabrique interdisciplinaire. Histoire et science politique, Rennes, Presses universitaires de Rennes, 2008, p. 191-203.

44 Marie-Emmnuelle Chessel, Le pouvoir de l'acheteuse. Des consommateurs catholiques en République (19001935), mémoire inédit présenté dans le cadre d'une habilitation à diriger les recherches, EHESS, 2009. 
Outre cette recherche d'éléments factuels donnant un sens à des concepts parfois vagues comme ceux d' " identité » ou d' " influence », prendre en compte la façon dont une institution se façonne et se légitime implique aussi de se confronter à de grandes questions de la culture politique française, notamment celle de la définition d'un intérêt général et de la représentation des intérêts particuliers, y compris dans sa reformulation invoquant la "société civile $\rangle^{45}$. Pour les aborder, il faut se demander d'où viennent les membres de l'institution, s'ils se considèrent comme les représentants d'intérêts précis - par ailleurs organisés ou non de façon formelle -, s'ils agissent comme tels, mais aussi s'ils font des efforts volontaires pour produire quelque chose qui relèverait d'un intérêt général - et par quelles procédures précises d'organisation des discussions, d'intervention d'experts « neutres », de vote... - ; enfin, si ce résultat est reconnu comme tel par d'autres acteurs. On rejoint là une question classique posée de la prosopographie : connaître les membres d'une institution et les intérêts particuliers qu'ils sont susceptibles de promouvoir, du fait de leur parcours antérieur ou de leurs cumuls de positions, suffit-il à comprendre les discours produits, suivant une démarche démystificatrice qui ne ferait finalement de l'institution qu'un voile pour cette somme d'intérêts? Sans exclure que telle puisse être la conclusion dans certains cas, prendre l'institution au sérieux implique de s'interroger sur les modes d'organisation collective, les raisons de s'engager, les discours qui peuvent ou non être explicitement tenus, ou encore les phénomènes de constitution de traditions internes, qui peuvent faire que l'institution soit, in fine, plus que la somme de ses membres.

Prendre au sérieux la question de la création et de l'identité des institutions n'implique pas pour autant de faire de celles-ci des blocs. En particulier, dès que l'on se confronte à une organisation particulière, se pose la question de l'échelle d'analyse, notamment lorsqu'on a affaire à quelque chose qui n'existe pas uniquement au niveau national, que ce soit une structure hiérarchisée sur une base au moins partiellement géographique (comme l'administration des Finances ou le Medef) ou une collection d'institutions locales relevant d'un même modèle, sans qu'une instance nationale les coiffe (comme les chambres de commerce). La question du degré d'autonomie des administrations ou organisations locales, voire de leur rôle dans l'élaboration de normes elles aussi locales, a été reposée à nouveaux frais par nombre de recherches récentes, notamment autour du syndicalisme patronal ou des conseils de prud'hommes ${ }^{46}$; elle se pose de façon très particulière à Paris, sans que l'on puisse postuler systématiquement une échelle d'action plus nationale qu'ailleurs. En outre, la répartition inégale sur le territoire des activités économiques peut très bien impliquer que des institutions a priori définies comme locales aient une influence bien plus large sur les règles propres à un secteur dominant localement : ainsi de la chambre de commerce de Lyon pour les mesures de qualité des textiles ou des institutions publiques ou groupes de pression du Bordelais ou de Bourgogne en matière de vins...

\section{Discours et pratiques : entrer dans les sources}

Si les historiens ont quelque chose à apporter ou à ajouter aux institutionnalismes des autres sciences sociales, c'est sans doute à la fois en termes d'études diachroniques, en particulier en portant attention aux moments de création institutionnelle, aux phénomènes de légitimation et d'installation dans la durée, et en matière de réflexion sur le traitement des

45 Pierre Rosanvallon, Le modèle politique français. La société civile contre le jacobinisme de 1789 à nos jours, Paris, Le Seuil, 2004 ; Claire Lemercier, «La France contemporaine : une impossible société civile ?», Revue d'histoire moderne et contemporaine, 52-3, juillet-septembre 2005, p. 166-179; Alain CнатRIOT, «La société civile redécouverte : quelques perspectives françaises ", Discussion Papers, WZB, SP IV, 2009-402.

46 Pierre Vernus (dir.), Les organisations patronales. Une approche locale (XIX $-X X^{e}$ siècles). Lyon: Cahiers du Centre Pierre Léon, 2002 ; Danièle Fraboulet, Quand les patrons s'organisent. Stratégies et pratiques de l'Union des industries métallurgiques et minières, Villeneuve-d'Ascq, Septentrion, 2007. 
sources. Lorsqu'il existe un fonds constitué comme celui d'une institution consultative - mais on pourrait dire à peu près la même chose des fonds d'organisations collectives, syndicats, corporations, voire partis politiques, et même, avec quelques variantes, de ceux des services administratifs ou d'une partie des fonds des tribunaux -, comprenant statuts, procédures de choix des membres, délibérations de commissions, sollicitations extérieures, documents de travail et rapports, il nous semble que certaines questions devraient se poser d'elles-mêmes. Elles impliquent de porter attention à la genèse des sources (celle de chaque écrit particulier, mais aussi de l'agencement que constitue un fonds): travailler sur des sources institutionnelles impose de réfléchir sur les techniques propres de l'institution et sur les pratiques des acteurs ${ }^{47}$. Ainsi, si des étapes comme la recherche des textes supposés créer l'institution puis encadrer son fonctionnement ou la reconstitution de listes de membres, et si possible d'informations prosopographiques, restent en général nécessaires, d'autres points d'entrée sont indispensables pour saisir les pratiques de l'institution, échapper à l'illusion juridique (celle du droit dans les textes), à l'illusion externaliste (celle de l'institution comme somme de ses membres) et à la pure monographie.

S'agissant des archives internes - listes de membres, procès-verbaux de réunions, rapports, jugements, circulaires... -, un savoir-faire encore peu formalisé est en train de s'élaborer, de recherche en recherche, pour les lire en comprenant à la fois les pratiques qu'elles peuvent refléter et les enjeux de leur mise en forme écrite et de leur conservation. Ainsi, les pratiques d'anonymisation, ou non, des intervenants dans les procès-verbaux se comprennent mieux lorsqu'on peut procéder à des comparaisons entre institutions (contemporaines ou non); les prendre en compte, comme d'autres codes ou éléments formels propres à ces sources, est crucial pour approcher tant l'image que l'institution veut donner d'elle-même que les pratiques d'élaboration d'un consensus. Plus généralement, les techniques d'écriture, de composition de formulaire, de reprographie... font partie des "investissements de forme » dont l'analyse permet de comprendre à la fois ce que les membres veulent faire et les moyens qu'ils emploient pour réaliser cette tâche. Dans le même ordre d'idées, ce que l'on peut saisir des moyens matériels de l'institution - budget, personnel, locaux - est important, moins pour mesurer une "importance » qui peut prendre d'autres figures que pour comprendre des éléments de permanence ou de précarité ou encore des liens de dépendance vis-à-vis d'autres organisations.

Cette attention portée notamment aux objets, ceux que l'on manipule en archives ou ceux dont parlent les archives, s'inspire des renouvellements de l'histoire des sciences ${ }^{48}$. Pour autant, elle ne doit pas empêcher, au contraire, d'avoir une lecture des textes qui ne s'en tienne pas à une histoire paresseuse des idées ou du droit. Pour ce faire, il est notamment important de cerner les formes du travail collectif de l'institution ${ }^{49}$ : plus ou moins hiérarchisé (qui tient la plume? qui apporte des éléments factuels pour les rapports ? qui participe réellement aux discussions ?), plus ou moins divisé en commissions ou sections par exemple. L'enjeu, qui peut sembler trivial, de l'affectation d'un ensemble de membres ou d'employés dans différents groupes, exclusifs ou non les uns des autres, parfois réduits, ou non, à une seule personne et plus ou moins pérennes, renvoie en réalité tant à des rapports de force propres à une situation précise qu'à de grandes questions politiques comme le rapport à

47 Pour un traitement exemplaire sur ce point, voir Igor Moullier, Le ministère de l'Intérieur sous le Consulat et le Premier Empire (1799-1814) : gouverner la France après le 18 brumaire, thèse de doctorat d'histoire sous la dir. de G. Gayot, Université de Lille III, 2004 (disponible en ligne).

48 Pour une transposition à l'étude d'une institution, voir Bruno Latour, La fabrique du droit. Une ethnographie du conseil d'État, Paris, La Découverte, 2002.

49 Voir ainsi, sur le rôle d'une catégorie d'acteurs trop longtemps négligée, Olivier DARD, Gilles RichARD dir., Les permanents patronaux : éléments pour l'histoire de l'organisation du patronat en France dans la première moitié du XX $X^{e}$ siècle, Metz, Centre de recherche histoire et civilisation de l'Université Paul Verlaine de Metz, 2005. 
l'expertise ou à l'intérêt général ${ }^{50}$. Parallèlement à cette attention aux procédures de production de discours et de décisions collectives, il est essentiel de prendre en compte le vocabulaire exact utilisé dans les sources, le langage apporté par chacun des acteurs et/ou celui produit collectivement par l'institution. Cela peut passer par des méthodes diverses, de la Begriffsgeschichte - l'histoire conceptuelle inspirée par l'historien allemand Reinhart Koselleck - à la lexicométrie, mais cela s'impose dans la mesure où le fait de nommer un problème politique ou de décider de la qualification juridique d'une situation est crucial pour la prise de décision.

Un terrain privilégié de l'étude fine des vocabulaires et des conflits sur les façons de nommer et de classer est le travail sur les controverses. C'est particulièrement le cas lorsqu'elles portent sur la création d'une nouvelle institution, supposée en remplacer ou en compléter une autre ou d'autres, suivant une idée importée de l'étranger ou d'un autre secteur, par exemple, constituent évidemment un terrain privilégié d'étude. En effet, elles permettent de travailler sur la notion, notamment popularisée par la micro-histoire, d'espace des possibles (qui considère que si certains chemins institutionnels sont très improbables à partir d'une situation donnée, l'avenir n'est pas pour autant totalement déterminé), tout en entrant en résonance avec les interrogations d'autres disciplines sur la variété des institutions empiriquement observées. Les périodes de reconstruction institutionnelle après une guerre ou une révolution, par exemple, représentent de ce point de vue un terrain d'observation privilégié ; il faut alors s'astreindre à suivre le destin des propositions longuement discutées mais abandonnées, des " échecs institutionnels », au moins autant que la généalogie des organisations effectivement mises en place. Cela en dit souvent plus sur ce qui était ou n'était pas possible, tant du point de vue du discours politique dominant - ainsi du tabou sur la représentation des intérêts particuliers dans la France d'après 1791 - que des pratiques les plus concrètes, les deux aspects étant d'ailleurs souvent liés - ainsi de la nécessité, si l'on supprimait les tribunaux de commerce, de payer un grand nombre de nouveaux juges fonctionnaires ${ }^{51}$.

Les controverses permettent aussi de mettre en évidence des formes variées de liens entre institutions (héritage ou opposition de modèles, consultation de l'une sur l'avenir de l'autre, mise en place de fronts communs, voire de comités de coordination...), qui n'épuisent pas, toutefois, la gamme des interactions et circulations possibles, des cumuls de fonctions ou trajectoires de membres à la demande mutuelle d'avis ou à l'échange d'informations, prévus par les textes ou mis en place spontanément. S'il est sans doute délicat d'étudier ainsi les institutions en se centrant sur leurs relations entre elles, voire de discuter de la notion de système d'institutions, avant d'avoir une connaissance minimale de chacune, les premiers acquis, sur certains terrains, de l'histoire des institutions économiques permettent déjà d'envisager une telle focale. En outre, même dans une démarche plus exploratoire, il est toujours utile de se poser ce type de questions pour éviter les illusions de centralité fondées sur l'immersion dans une seule source. Ainsi, bien qu'il n'existât alors que peu de travaux sur le conseil municipal et sur le tribunal de commerce de Paris, en bonne partie pour des raisons d'accès aux sources, étudier leurs liens de différents types (ainsi que ceux de la Banque de France, bien mieux connue) avec la chambre de commerce de Paris a notamment permis de mettre en évidence l'association changeante de cette dernière tantôt avec le monde de la haute banque, tantôt avec des formes de représentation des secteurs de l'économie parisienne, ainsi que, plus globalement, de comprendre le rapport des élites économiques à la notion de «fonctions gratuites », clé de leur implication institutionnelle ${ }^{52}$. De simples listes comparées

50 Pour un développement plus détaillé sur ce point, voir Alain ChатRIot, Claire Lemercier, « Une histoire... », art. cit.

51 Antoine VAuchez, Laurent Willemez, La justice face à ses réformateurs (1980-2006), Paris, PUF, 2007.

52 Claire Lemercier, «Liens privés et régulation de l'économie : la famille et l'institution (Paris, XIXe siècle) », Revue d'histoire du XIX ${ }^{e}$ siècle, $\mathrm{n}^{\circ} 33-2,2006$, p. 23-53. 
de membres et mentions mutuelles dans les correspondances ou les procès-verbaux donnent déjà des éléments très importants pour situer ainsi une institution par rapport à d'autres.

\section{En guise de conclusion...}

Nombre d'objets de l'histoire économique auraient pu illustrer le développement des approches à l'échelle des institutions. En voici, pour conclure, quelques exemples plus ou moins détaillés, qui illustrent souvent l'importance des circulations interdisciplinaires et du travail collectif pour de telles recherches.

Le premier concerne l'histoire de la protection sociale. Depuis longtemps déjà, l'histoire de l'État-providence n'est pas étudiée comme un bloc : historiens, sociologues et politistes se montrent particulièrement attentifs aux institutions qui ont préparé les réformes sociales ou permis leur application. Au cœur de la «nébuleuse réformatrice » repérée autour de $1900^{53} \mathrm{se}$ trouvent bon nombre d'institutions, dont la plus célèbre reste sans doute le Musée social ${ }^{54}$. Mais étudier les développements ultérieurs nécessite aussi de se pencher précisément sur des institutions. On ne peut suivre l'histoire complexe des assurances sociales dans la France des années 1920 et 1930 sans étudier le fonctionnement des différentes caisses ${ }^{55}$. On ne peut comprendre, au-delà des proclamations rhétoriques, le fonctionnement de la politique de la famille du régime du maréchal Pétain sans en suivre l'ensemble des rouages à des échelles où cette politique est mise en place via des administrations et des organisations ${ }^{56}$. De même, cette histoire ne peut rester franco-française, mais doit tenir compte du rôle des institutions internationales, comme, au $\mathrm{XX}^{\mathrm{e}}$ siècle, la place éminente tenue par le Bureau international du travail ${ }^{57}$.

Un deuxième exemple, qui concerne un domaine seulement émergent de recherche, concerne la concurrence. L'approche interdisciplinaire, aujourd'hui assez rebattue, des « districts industriels $»^{58}$, vise au départ à élucider ce qui est décrit comme un paradoxe : le constat de formes de coopération entre entreprises semblant « objectivement » en situation de concurrence particulièrement vive. Le caractère paresseux des réponses apportées à cette question en termes de simple proximité spatiale ou de particularités culturelles locales a conduit très récemment à mettre plutôt l'accent sur le fonctionnement concret des normes et des institutions, souvent locales, permettant cette coopetition, notamment les organisations locales de branches, mais aussi les cartels ou ententes regroupant plutôt de grandes entreprises $^{59}$. Si une approche institutionnelle peut ainsi permettre de sortir de descriptions idylliques d'une coopération naturelle, réciproquement, la concurrence peut aussi être vue comme une construction institutionnelle : puisqu'elle n'est jamais un simple état de nature, les politiques de la concurrence comme les structures mises en place pour les contourner mérite

53 Christian Topalov (dir.), Laboratoires du nouveau siècle. La nébuleuse réformatrice et ses réseaux en France (1880-1914), Paris, éditions de l'EHESS, 1999.

54 Janet Horne, Le Musée social. Aux origines de l'Etat providence, Paris, Belin, 2004.

55 Michel Dreyfus, Michèle Ruffat, Vincent Viet, Danièle Voldman, avec la collaboration de Bruno Valat, Se protéger, être protégé. Une histoire des Assurances sociales en France, Rennes, Presses universitaires de Rennes, 2006.

56 Christophe Capuano, Vichy et la Famille. Réalités et faux-semblants d'une politique publique, Rennes, Presses universitaires de Rennes, 2009.

57 Paul-André Rosental, « Géopolitique et Etat-providence. Le BIT et la politique mondiale des migrations dans l'entre-deux-guerres », Annales. Histoire, Sciences sociales, 61, 1, janvier-février 2006, p. 99-134.

58 Pour une revue critique stimulante en français, voir Jean-Claude DAumAs, « Districts industriels : du concept à l'histoire », Revue économique, vol. 58, $\mathrm{n}^{\circ} 1$, janvier 2007, p. 131-152.

59 Alain Снатвіот, «Les ententes : débats juridiques et dispositifs législatifs (1923-1953). La genèse de la politique de la concurrence en France », Histoire Economie et Société, 1, 2008, p. 7-22 ; Claire Lemercier, «Looking for 'industrial confraternity'. Small-scale industries and institutions in 19th-century Paris », Enterprise \& Society, 10-2, juin 2009, p. 304-334. 
leur étude - encore moins avancée en France, toutefois, que celle des organisations se confrontant aux États-Unis aux lois anti-trusts.

Certaines questions économiques transversales nécessitent des approches plurielles, qui peuvent justifier le choix d'une entrée institutionnelle. Pour travailler sur l'organisation scientifique du travail dans l'Europe de l'entre-deux-guerres, le Bureau international du travail constitue un observatoire privilégié ${ }^{60}$. Pour saisir la place des inventeurs et de l'innovation dans la France de la Belle époque, loin de la vision romantique du génie solitaire, c'est bien à des institutions que l'historien doit se confronter - des académies aux tribunaux, en passant par les associations d'inventeurs ou les commissions parlementaires ${ }^{61}$. S'interroger sur la productivité dans la France des années cinquante est possible à partir d'une entrée biographique, mais celle-ci est aussi une invitation à suivre l'ensemble des institutions pratiquées par cet acteur, ce qui permet de retrouver la plupart des autres figures de la modernisation de la France - dans un système d'institutions construit par leurs carrières complexes $^{62}$. Enquêter sur les choix en matière d'Europe économique au début du marché commun peut paraître relever de la diplomatie et des décisions politiques au sommet du pouvoir; or, c'est aussi suivre des acteurs collectifs mobilisés via des institutions diverses ${ }^{63}$. Enfin, reconstituer un fait divers de la France des années cinquante partant d'une histoire d'empoisonnement permet de restituer tout un monde social et économique et passe par l'étude précise d'une institution comme l'ONIC, l'Office national interprofessionnel des céréales, qui joue un rôle clef dans la régulation du marché du blé et de la farine dans la France du $X^{\mathrm{e}}$ siècle, pendant plus de cinquante ans ${ }^{64}$. Ainsi, que l'on soit amené à l'institution par la recherche archives, de points d'observation un peu stables, par la volonté de situer un acteur ou un événement ou de suivre une prise de décision, des approches qui ont un air de famille s'imposent à propos d'objets très variés. Différents questionnements ont ainsi mené à mettre l'accent non seulement sur la nécessité de ne pas oublier les institutions, sur le fait qu'elles pouvaient influencer différents phénomènes économiques, mais aussi sur l'importance d'une étude de leurs pratiques (des plus matérielles à la création de catégories statistiques ou juridiques) et des différents groupes d'acteurs qui les font vivre ou tentent de les utiliser.

60 Thomas CAYET, «Travailler à la marge : le Bureau International du Travail et l'organisation scientifique du travail (1923-1933)», Le Mouvement Social, n²28, septembre 2009, p. 39-56.

61 Gabriel Galvez-Behar, La République des inventeurs. Propriété et organisation de l'innovation en France (1791-1922), Rennes, Presses universitaires de Rennes, 2008.

62 Régis Boulat, Jean Fourastié, un expert en productivité. La modernisation de la France (années trente années cinquante), Besançon, Presses universitaires de Franche-Comté, 2008

63 Laurent W ARLOUZET, Quelle Europe économique pour la France? La France et le marché commun industriel 1956-1969, thèse de doctorat d'histoire sous la direction d'E. Bussière, Université Paris IV, 2007.

64 Stephen L. Kaplan, Le pain maudit. Retour sur la France des années oubliées 1945-1958, Paris, Fayard, 2008. 